\title{
CUIDADO INFORMAL, UN RETO ASUMIDO POR LA MUJER
}

\author{
INFORMAL CARE CHALLENGE ASSUMED BY WOMEN
}

\author{
SANDRA VAQUiro RodrígueZ* \\ JASNA STIEPOVICH BERTONI ${ }^{* *}$
}

\begin{abstract}
RESUMEN
La familia constituye la primera institución que genera cuidados en situaciones de dependencia, donde es la mujer la que proporciona cuidados en forma invisible y continua. Este traslado de responsabilidades del cuidado de la salud desde el estado a la familia, es necesario que se visibilice como problemática social, se establezcan políticas con enfoque de género que determinen correcciones de inequidades que proporcionan los estereotipos culturales tradicionales en el trabajo doméstico, al igual que se visibilice la necesidad de una mayor intervención de enfermería como apoyo al cuidado informal. En este artículo se realizó una revisión bibliográfica acerca del cuidado informal, en lo que respecta a la inequidad de género en el cuidado de la salud en Chile. Se observa la feminización en el cuidado informal como un paradigma de desventajas, esfuerzos, sacrificios relativos al género que conllevan a desigualdades innecesarias, evitables e injustas. La necesidad emergente de implementar estrategias desde todos los ámbitos: políticos, sociales, sanitarios y culturales al igual que generar conocimientos para el desarrollo de la ciencia de Enfermería, evidenciando el cuidado informal como una continuación del cuidado en el ámbito privado con contribución económica invisible del sistema de salud. Se proponen conductas de autocuidado en relación al riesgo en la salud de la cuidadora, dada la sobrecarga de cuidar y la importancia para enfermería en cuanto al saber, el ser y el hacer en el cuidado.
\end{abstract}

Palabras clave: Cuidado informal, género, inequidad, atención de enfermería.

\begin{abstract}
The family is the first institution to generate dependency care in situations where it is the woman who provides invisibly and continuous care. This transfer of responsibilities of health care from the state to the family, it is necessary to make visible as social problems, policies are enacted with gender inequities to identify corrections that provide traditional cultural stereotypes in domestic work, as is highlight the need for increased nursing intervention to support informal care. In this paper, we review the literature on informal care in regard to gender inequity in health care in Chile. This enables conclude that there is the feminization of informal care as a paradigm of disadvantages, efforts, sacrifices on the gender inequalities that lead to unnecessary, avoidable and unfair. The emerging need to implement strategies from all aspects: political, social, health and cultural as well as generate knowledge for the development of the Science of Nursing Care showing a continuation of informal care in the private sphere invisible economic contribution of the health system. Self-care behaviors are proposed in relation to risk the health of the caregiver, given the burden of care and the importance for nursing as knowing, being and doing in care.
\end{abstract}

Key words: Informal care, gender, inequity, nursing care.

Fecha recepción: 15/12/08 Fecha aceptación: 21/06/10

\footnotetext{
*Enfermera, candidata a grado Magíster en Enfermería Universidad de Concepción. Concepción, Chile. E-mail: sandravaq@hotmail.com

${ }^{* *}$ Doctora en Enfermería, docente programa de doctorado Universidad de Concepción. Concepción, Chile. E-mail: jstiepov@udec.cl.
} 


\section{INTRODUCCIÓN}

El presente trabajo tiene por objeto visibilizar la inequidad de género en el cuidado informal, donde es la mujer quien ejerce esta actividad invisible y exigente dentro del sistema del cuidado de la salud. Su importancia radica en el aumento de la demanda del cuidado solicitado, teniendo en cuenta el cambio demográfico: aumento de la longevidad y la expectativa de vida; adicionando a ello el cambio de la familia monoparental, donde es la mujer en muchas oportunidades jefe de hogar siendo no sólo la responsable del cuidado de la salud de la familia sino asumiendo el costo que éste genera. Las mujeres cuidadoras como un grupo vulnerable tienen derecho a una mejor calidad de vida, a una disminución en la sobrecarga del cuidar y en el riesgo para su salud que éste significa.

El sistema de salud ha evolucionado de un modelo paternalista, donde el clínico, según su juicio, creía poder interpretar las preferencias de sus usuarios a un modelo de decisión informada, al usuario, donde el profesional expone las opciones disponibles y él decide la alternativa que considere se adapte a sus expectativas. Situación que no deja de tener un riesgo, dado que la participación del cuidado formal no ha sido muy extensiva y la solicitud de apoyo desde la comunidad ha sido desplazada a otras instancias.

La mujer sobrerrepresentada en el rol de cuidadora informal asume la continuidad del cuidado de la salud en el interior del hogar, en el ámbito de lo privado en forma inequitativa, es por ello que se pretende investigar esta temática y propiciar la creación de programas específicos dirigidos a las cuidadoras, asignación de recursos, incorporación del enfoque de género en lo que compete al cuidado de personas enfermas o dependientes en el hogar; la necesidad del apoyo formal y un mayor cuidado e intervención de enfermería.

\section{Generalidades del cuidado}

El cuidar es un acto inherente a la vida, es el resultado de una construcción propia de cada situación de promover, proteger y preservar la humanidad; en este contexto, la salud es asumida por la mujer en un medio de marginalidad de vínculos familiares, culturales y sociales de la forma como se concibe el cuidado (1).

El cuidado existe desde el comienzo de la vida; el ser humano como todos los seres vivos ha tenido siempre la necesidad de ser cuidado, porque cuidar es un acto de vida, que permite que la vida continúe. Las personas necesitan atenciones desde que nacen hasta que mueren, por tanto cuidar es imprescindible para la vida y para la perpetuidad del grupo social (2). El fenómeno del envejecimiento poblacional, los procesos de transición demográfica, epidemiológica y el incremento de la longevidad mundial, tienen impacto en la solicitud del cuidado y modifican la demanda en salud, promueve las desventajas sociales, económicas; factor al que deberá añadírsele una mejoría de la calidad de vida, específicamente, porque relaciona el aumento del índice de dependencia de las condiciones de salud física, mental y la reducción en años esperados de vida activa y saludable, así como un aumento creciente de enfermedades crónicas más que agudas, y más bien progresivas que regresivas (3). Chile se encuentra en un periodo de transición demográfica y epidemiológica avanzada, centrando cada vez más la morbilidad y mortalidad en enfermedades crónicas no transmisibles del adulto, favoreciendo la necesidad de cuidado informal al interior de las familias (4).

Sin duda, la familia es la mayor prestadora de atención de salud en nuestro medio, es una de las instituciones sociales más antiguas y más fuertes, considerada como un sistema y una unidad cuya socialización e interacción mutua afecta en mayor o en menor grado a cada uno de sus miembros; donde uno 
de ellos asume el rol de cuidadora/or principal en caso de discapacidad o limitación. La función social, educativa, laboral y de poder de la familia ha sido determinante en la perpetuación del rol de cuidadora asumido por la mujer, en su gran mayoría en calidad de esposa, hija o madre; rol asignado desde la división sexual del trabajo cuya organización está centrada en diferencias de género; rol reproductivo de la mujer basado en relaciones del afecto y actividades domésticas, dentro de la estructura familiar y rol productivo en el hombre y hegemónico en la dinámica económica familiar (5).

Dentro de la experiencia del cuidado, se ha podido establecer que el diario vivir de un adulto con enfermedad crónica genera un impacto en la vida cotidiana en las personas y las familias que cuidan, teniendo en cuenta el agobio humano de tener que enfrentar la muerte, innumerables situaciones de difícil manejo, toma de decisiones, competencia entre el cuidar y las metas de vida personal, ocasionando cambios en los roles que afectan la calidad de vida, el sueño, descanso, actividad social, emocional, económica y laboral, siendo de mayor complejidad en casos de dependencia, postrados (6).

El cuidado informal se ha definido como los cuidados proporcionados por familia, amigos, vecinos u otras personas al interior del hogar en un mundo privado, a personas ancianas, enfermas y dependientes; fundamentadas en relaciones afectivas, de parentesco "asuntos familiares" y de "género", rasgos que caracterizan y afectan su visibilidad y reconocimiento social. Por tanto, no reconocido como un trabajo remunerado sin precio en el mercado, que se confunde con una carencia de valor, sin límites conocidos de tiempo (7). El aporte económico de la mujer ha sido subestimado, la conciencia colectiva cultural establece la vinculación del "cuidado" a algo maternal, asociándolo al rol reproductivo. Por tanto, debe darse como algo natural; esta invisibilidad del género femenino dificulta su reconocimiento en el cuidado informal y el cambio hacia un nuevo paradigma (8).

\section{Cuidado informal y género: inequidad y feminización del cuidado}

El llamado enfoque de género en salud se visualiza a mediados del siglo XX. El término género pone de manifiesto los comportamientos culturales, sociales y asignación de roles que diferencian la forma en que la sociedad construye el "ser hombre" o "ser mujer," no como distintos sino como desiguales. El concepto de género es relacional, revela la inequidad de las relaciones sociales desde un principio de poder y desigualdad centradas en carencias, exclusiones de ingresos y participación innecesarias, evitables e injustas (9).

El inicio de la invisibilidad de la cuidadora informal se da a partir de la asignación del rol de cuidadores en el contexto socio-cultural de mujeres y hombres sobre sus actitudes y conductas; donde el cuidado es algo inherente a toda cultura humana y es la mujer a lo largo de la historia responsable por la salud física y mental de la familia en el proceso salud y enfermedad (10). La carga diferenciada en la distribución del papel de cuidadores entre hombres y mujeres y la presencia del género femenino en el cuidado es un hecho; aunque ello no significa que el cuidado sea realizado en exclusividad por mujeres. Sin embargo, el cuidado asume una connotación de feminización e inequidad donde el perfil típico de las personas que asumen el papel de cuidadora principal es el de una mujer sin empleo, de menos nivel educativo, responsable de las tareas domésticas, de clase social baja, familiar directo y quien convive con la persona que cuida, ejerce actividades de prevención como de cuidado respecto a la salud familiar y no sólo cuida, sino que apoya y ayuda a otras mujeres en el cuidado (11). La mujer asume el cuidado como un compromiso moral, natural, marcado por el afecto, socialmente a un costo alto, definido como 
responsabilidad, tarea impuesta, deber sancionable no valorada, ni remunerada, hasta el momento en que estos cuidados no son asumidos. Mientras que en el género masculino el cuidado se da como una opción. Lo que resalta la existencia de una diferente valoración social respecto al desarrollo de estas tareas y el sesgo de género que esto supone; a pesar de ello, y al valioso aporte del género femenino dentro del sistema de salud, el ejercer la función intermediaria entre el enfermo dependiente y los sistemas sanitarios son invisibles, únicamente se hacen evidentes cuando estos cuidados no son asumidos (12). La mujer, cuidadora por excelencia, aporta un invisible, importante e invalorado rol como agente de salud, como cuidadora informal para su familia y para la sociedad, es ella quien toma decisiones relacionadas con el cuidado y asume además multiplicidad de roles (13).

La atención informal en salud plantea por tanto dos desigualdades relacionadas con la responsabilidad del cuidado: las cargas diferenciales del cuidado entre hombre y mujer, y entre la familia y el Estado, las cuales requieren ser planteadas como elementos esenciales y factores críticos de debate dentro de la asignación de recursos y creación de nuevos programas que promuevan reformas en los servicios de salud y en las políticas económicas y sociales de un país (7).

Se percibe que en los últimos años el Estado ha estado gradualmente transfiriendo a las familias la responsabilidad del cuidado de personas dependientes, vinculadas a procesos de salud tanto crónicos como agudos en las diferentes etapas del ciclo vital, siendo más evidente en personas senescentes. La provisión de cuidados se manifiesta con pequeños sacrificios, sin ningún reconocimiento social y económico, atribuido sin ser parte, a la esfera de lo privado, siendo un problema que debe formar parte de derechos ciudadanos y democráticos (14).

La pérdida del equilibrio del cuidado nos lleva a reflexionar en el contexto de derecho y deber cuidar, justicia de oportunidades y valorización del trabajo, dentro de un concepto de desventajas entre hombres y mujeres, familias y Estado; evaluando la distribución de poder, recursos y responsabilidades. Cambiar el protagonismo inequitativo por un nuevo enfoque participativo, cultural, social neutro e imparcial del cuidado compartido que involucra a todas(os).

Comprende además algunos valores como el tiempo dedicado al cuidado, la intensidad, y oportunidad, acotando que las mujeres con un bajo nivel de ingresos y elevada edad dedican más horas al cuidado informal no remunerado, contribuyendo al mantenimiento de este sistema de bienestar (11). Mientras que los varones ocupados o no ocupados con un bajo nivel de ingreso tienen menor probabilidad de desempeñar este tipo de labor informal, lo que sugiere mayor grado de división sexual del trabajo, relacionado ello con las escasas oportunidades laborales de las mujeres en los grupos de población de menos ingreso (15).

Considerando la importancia del cuidado informal, aún no existen intervenciones efectivas que produzcan transformaciones educativas, culturales de los derechos sociales y laborales de la mujer. $\mathrm{Ni}$ acciones que compatibilicen la responsabilidad familiar y la del Estado; ni reconocimientos mediante contratos de trabajo y seguros de dependencia, ni creación de programas a las (los) cuidadoras/res, ni asignación de recursos económicos acorde a las reales necesidades de cuidado (15).

\section{Cuidadora(or) informal y la salud en Chile}

La Organización Panamericana de la Salud (OPS) incorpora la perspectiva de género en la salud como un constructo de carácter ético, resaltando la realidad en las relaciones de desigualdad entre hombres y mujeres, que permiten visibilizar inequidades de carácter económico, social y laboral (16).

Chile ha evolucionado desde un sistema 
fundamentalmente público hacia uno de carácter mixto, con un importante componente público y privado. El componente público, Fondo Nacional de Salud (FONASA), adscribe la población de menos ingresos y con más alto riesgo, y uno privado, Instituciones de Salud Previsional (ISAPRE), atrae a la población joven y según su capacidad de ingresos puede comprar planes de salud con mejores coberturas, a un mayor costo para las mujeres y los adultos mayores. Por tanto, se establece una correlación entre el nivel de ingreso y acceso a los servicios, teniendo en cuenta que las mujeres, especialmente las más pobres, consultan más que los hombres, es decir las mujeres tienen mayor demanda, mayor necesidad en salud y tiene mayor percepción de la enfermedad (17).

El hecho que el cuidado informal sea un trabajo físico, emocional en un escenario de inequidad de género, en el cual se ejercen múltiples roles simultáneos de madre, esposa, trabajadora y el desarrollo de roles propios del cuidado, traduce el impacto de cuidar en los diferentes aspectos de la salud y de calidad de vida, se vuelve un factor de riesgo para su salud; adicional a ello tiene un alto costo de oportunidades, ya que, entre otros, se puede perder o tener que renunciar a la actividad laboral. La vida de la cuidadora se acondiciona por su papel, el $68 \%$ de las cuidadoras percibe que cuidar afecta de manera importante la salud, su vida social y personal (18). Sobre su propia salud, se encuentran a menudo cansadas del cuidado en un 51,2\% de los casos, el 32,1\% se siente deprimida. Sobre su vida afectiva, el $64,1 \%$ declara que se han visto obligadas a disminuir su tiempo de ocio. Sobre aspectos laborales: el 26\% confiesa no poder plantearse trabajo fuera; el $11,5 \%$ ha tenido que abandonar el trabajo, el $12,4 \%$ se ha visto obligada(o) a reducir su jornada de trabajo (18).

Los efectos que puede tener el cuidado en la vida del cuidador como resultados adversos que pueden afectar su calidad de vida, salud física, aspectos sicológicos, y en su vida social están relacionados con el cambio que se genera en la vida del cuidador (19); el impacto que tiene cuidar ha sido definido como efectos de sobrecarga en el cuidador, valorada con la escala de Zarit (20), el cual se relaciona directamente con el tiempo de dedicación al cuidado de gran intensidad y de larga duración (11). Se ha estudiado algunas estrategias preventivas en relación a la sobrecarga y cansancio del cuidador en consideración a que los cuidadores que presentan cansancio emocional o mental tienen mayor riesgo de morir. La North American Nursing Diagnosis Association (NANDA) ha enfocado el diagnóstico de enfermería hacia tres aspectos fundamentales: la alteración del estilo de vida del cuidador familiar, el bienestar del cuidador y la ejecución del rol; resueltos a través de intervenciones de apoyo, cuidados intermitentes, y el aumento de afrontamiento y enseñanza individual (21).

Diferentes estudios muestran que las mujeres cuidadoras presentan casi dos veces más sobrecarga que los hombres cuidadores, y las cuidadoras más jóvenes presentan más sobrecarga que las de más edad (11). Sumado a lo anterior, la sobrecarga lleva a menos descanso, menos horas de sueño, menos actividades recreativas, afectando la salud mental, e incrementa los riesgos la salud cardiovascular.

Algunos autores incorporan el término calidad de vida relacionada con la salud, donde los cuidadores de ambos sexos perciben una peor salud en comparación con las personas no cuidadoras/es. El riesgo de mala salud mental y física aumenta en los cuidadores con respecto a los no cuidadores, con un impacto negativo mayor de cuidar en las mujeres, evidenciando cantidad de cuidados y el riesgo de deterioro de la salud, lo que sustenta la necesidad imperiosa de requerimiento de apoyo social de la familia, amigos, vecinos, voluntarios, asociaciones del Estado y de las instituciones formales (22). Las medidas de soporte y la atención a los cuidadores requieren que uno de los servicios priori- 
tarios en el sistema sanitario sea la atención domiciliaria como función básica de la atención primaria; en especial apoyo e información del profesional de enfermería (23). Se considera que el soporte que éstos tengan es un importante factor de estabilidad emocional y de calidad de vida (18).

Una investigación de cuidados de pacientes con SIDA en el hogar concluye que el apoyo social es más importante que la necesidad de conocimiento en un grupo, donde la enfermera tiene gran importancia (24). Sin embargo, no se debe dejar de visualizar que el sistema de salud no ofrece en muchas oportunidades el apoyo formal para acompañar e informar sobre las opciones disponibles de un determinado problema; existen situaciones críticas en salud de las mujeres cuidadoras informales del adulto mayor postrado que obligan a enfrentar decisiones que le implican cambio en el estilo de vida y a decidir con insuficiente información, escasa participación y apoyo por parte de los cuidadores formales en salud (25).

\section{Autocuidado en la cuidadora}

Finalmente podríamos preguntar, ¿cómo promover y apoyar el autocuidado en las cuidadoras para mejorar su calidad de vida desde la intervención de enfermería? Cuestionamiento que permite definir el autocuidado como la práctica de actividades aprendidas que los individuos realizan en favor de sí mismos para mantener la vida, la salud y bienestar. Acciones deliberadas, aplicadas de forma voluntaria en el tiempo y aprendidas en la familia (26). Resalta la importancia de la teoría de enfermería de Orem que articula el autocuidado, déficit de autocuidado y el sistema enfermero a través de acciones de educación y promoción en la vida diaria para fortalecer el autoconcepto, mantener la motivación por sí y para sí mismo junto con el trabajo conjunto de acompañamiento enfermera para evitar el déficit de autocuidado (26), el cual sigue siendo un problema difícil de enfrentar en cuanto a la capacidad del individuo de comprometerse con su autocuidado y lograr agencia de autocuidado en la/el cuidadora/or para cuidarse, cuidar y ser cuidado como acto individual y de reciprocidad (27).

El proceso educativo permite compartir experiencias y conocimientos. Por tanto, se hace una herramienta fundamental de participación dentro del proceso de cuidado, con actividades de autocuidado como un comportamiento social activo, que promueve la calidad de vida (28).

\section{CONSIDERACIONES FINALES}

Hay un desplazamiento de cuidados cada vez más complejos hacia el sistema informal, alta de atención hospitalaria, programas de cirugía ambulatoria, reformas de atención siquiátrica, personas con enfermedades graves dependientes, o en situación terminal; los servicios formales participan de manera minoritaria en el cuidado de las personas dependientes que viven en la comunidad. Esta mayor demanda por atenciones en salud derivada de mayores necesidades, nos lleva al desafío de intervención y apoyo de enfermería en las acciones de educacion, prevención y fomento de autocuidado. Los límites del cuidado son difíciles de enmarcar en cuanto a qué se hace, a quién, dónde y durante cuánto tiempo (11).

La asimetría entre la economía productiva y reproductiva, la distribución de cargas al interior del hogar, la responsabilidad de la mujer de asegurar la salud familiar, refleja las condiciones de desigualdad entre el trabajo doméstico y laboral.

El cuidado informal, entendido como una extensión del cuidado de la salud en personas dependientes, requiere se establezca un programa dirigido a las cuidadoras(es) dentro de las políticas del Estado con enfoque de género -al ser realizado en su mayoría por 
mujeres de nivel socioeconómico bajo, bajo nivel educativo y algún grado de parentescoque evite el impacto negativo que el cuidar tiene en la salud del cuidador (7).

Se requiere que se reconozca el cuidado informal como un trabajo que aporta a la economía, no como un recurso sin valor con el que se cuenta normalmente y que se asigna dentro del cuidado doméstico no pagado; teniendo en consideración el creciente aumento de la demanda del cuidado, el cuestionamiento de la disponibilidad futura de las cuidadoras informales, las familias monoparentales y las reformas de los sistemas de atención en salud (8).

Realizar intervención de enfermería en el cuidado del individuo y de la familia, proporcionando conocimiento y habilidades para afrontar el cuidado en las mejores condiciones posibles; técnicas de relajación, manejo de estrés, organización del tiempo y estrategias para compartir el cuidado con otros miembros de la familia. Promover un entorno mental, físico, espiritual, de soporte de protección y recuperación, dado con la creación de asesorías domiciliarias con un grupo interdisciplinario asignado a las cuidadoras (18).

Implementar un nuevo paradigma en la incorporación del hombre al trabajo doméstico como evento revolucionario que se requiere en los nuevos roles socioculturales, con participación equitativa hombre/mujer, con resultados reflejados en la dinámica familiar de igualdad y respeto en la socialización de los hijos.

\section{REFERENCIAS}

1. Reyes Luna J, Jara Concha P, Merino Escobar JM. Adherencia de las enfermeras/ os a utilizar un modelo teórico como base de la valoración de enfermería. Cienc. Enferm. 2007; 13 (1): 45-57.

2. Herrera E. Cuidadoras y cuidadores de personas dependientes y enfoque de género en Chile. Departamento de Programas y proyectos. Gobierno de Chile. Fonadis. 2007.

3. Bayarre Vea H, Pérez Piñero J, Menéndez Jiménez J. Las transiciones demográfica y epidemiológica y la calidad de vida objetiva en la tercera edad. GEROINFO. 2006; 1 (3): 1-20.

4. Szot M J. Análisis epidemiológico de la mortalidad por tumores sólidos en la Región Metropolitana, Chile. Rev.Med. Chile. 20003; 131 (6): 641-649

5. Siles Gonzáles J, Solano Ruiz C. Estructuras sociales, división sexual del trabajo y enfoques metodológicos: la estructura familiar y la función socio-sanitaria de la mujer. Invest. Educ. Enferm. 2007; 25 (1):66-73.

6. Pinto Afanador N. La cronicidad y el cuidado familiar, un problema de todas las edades: los cuidadores de adultos. Avanc. Enferm. 2004; 22 (1): 54-60.

7. García-Calvente MM, Mateo-Ramírez I, Eguiguren AP. El sistema informal de cuidados en clave de desigualdad. Gac Sanit. 2004; 18 (supl 1):132-139.

8. Rodríguez Camarero ML, Rodríguez Camarero N, Azanon Hernández R, Torres López A, Rodríguez Salvador MM, Muñoz Ronda F. Mujeres, salud y cuidados familiares: Instituciones económicas desde la perspectiva antropológica. Index Enferm. 2007; 16(58):45-49.

9. Borrell C, García-Calvente MM, MartiBosca JV. La salud pública desde la perspectiva de género y clase social. Gac Sanit. 2004; 18 (supl 1):2-6.

10. Pezo Silva MC, Souza Praca N, Costa Stefanelli M. La mujer responsable de la salud de la familia. Index Enferm. 2004; 13(46):13-17.

11. García-Calvente MM, Mateo-Rodríguez I, Maroto-Navarro G. El impacto de cuidar en la salud y en la calidad de vida de las mujeres. Gac Sanit. 2004; 18 (supl 2):83-92. 
12. Valderrama Ponce MJ. El cuidado, ¿una tarea de mujeres? Vasconia. Cuadernos de Historia-Geografía. 2006; 35: 373385.

13. Valenzuela S, Paravic T, Sanhueza O, Stiepovich J. Programa de salud de la mujer en Chile: Relación entre lo teórico y lo empírico en la comuna de Concepción. Cienc. Enferm. 2001; 12 (1): 80-91.

14. Aguirre R. Familias como proveedores de servicios de cuidados. Documento preparado para los debates on line. Hallado en: www.e-cofi.net/fichero. php?id=29\&zona=-1-España [acceso en 30/10/08].

15. La Parra D. Contribución de las mujeres y los hogares más pobres a la producción de cuidados de salud informales. Gac Sanit. 2001; 15 (6): 498-505.

16. Gómez Gómez E. Equidad, género y salud: Retos para la acción. Panam salud pública. 2002; 11 (5-6): 454-461.

17. Vega J, Bedregal P, Jadue L, Delgado I. Equidad de género en el acceso a la atención de salud en Chile. Rev. Méd. Chile. 2003; 131 (6): 669-678.

18. Torres Egea P, Ballesteros Pérez E, Sánchez Castillo PD, Gejo Bartolomé A. Programas, intervenciones y redes de apoyo a los cuidadores informales en salud. Nursing. 2008; 26 (6): 56-61.

19. Giraldo Molina CI, Franco Agudelo GM. Calidad de vida de los cuidadores familiares. Aquichan. 2006; 1(6): 38-53.

20. Zarit SH, Reever KE, Bach-Peterson J. Relatives of the impaired elderly: correlates of feelings of burden. Gerontologist. 1980; 20: 649-55.

21. Péculo Carrasco JA, Rodríguez Bouza M, Casal Sánchez MM, Rodríguez Ruiz HJ,
Pérez Santos de los OC, Martín Tello JM. Cansancio del cuidador informal en la asistencia sanitaria urgente extrahospitalaria. Tempos Vitales. 2006; 6 (1): [ $\sin$ número].

22. Larrañaga I, Martín U, Bacigalupe A, Begiristáin JM, Valderrama MJ, Begoña A. Impacto del cuidado informal en la salud y la calidad de vida de las personas cuidadoras: Análisis de las desigualdades de género. Gac Sanit. 2008; 22 (5): 443-50.

23. Ubiergo Ubiergo MC, Regoyos Ruiz S, Vico Gavilán MV, Reyes Molina R. El soporte de enfermería y la claudicación del cuidado informal. Enferm Clin. 2005; 15 (4): 199-205.

24. Cazenave GA, Ferrer S-GX, Castro BS, Cuevas AS. El familiar cuidador del paciente con SIDA y la toma de decisiones en salud. Rev. Chil. Infectol. 2005; 22 (1): 51-57.

25. Mendoza PS, Jofré AV, Valenzuela SS. La toma de decisiones en salud y el modelo conceptual de Ottawa. Invest. Educ. Enferm. 2006; 24(1): 86-92.

26. López Díaz AL, Guerrero Gamboa S. Perspectiva internacional del uso de la teoría general de Orem. Invest. Educ. Enferm. 2006; (24) 2: 90-100.

27. Velandia Arias A, Rivera Álvarez LN. Confiabilidad de la escala Apreciación de la agencia de autocuidado (ASA), segunda versión en español, adaptada para población colombiana. Av. Enferm. 2009; 27(1): 38-47.

28. Zea Herrera MC. La experiencia del aula universitaria de mayores: Enseñanzaaprendizaje de cuidado y autocuidado. Invest. Educ. Enferm. 2009; 27(2): 244253. 